\title{
ENDPOINT ESTIMATES FOR MULTILINEAR FRACTIONAL INTEGRALS
}

\author{
LIN TANG \\ (Received 11 June 2004; accepted 3 February 2007) \\ Communicated by A. H. Dooley
}

\begin{abstract}
We study the boundedness for multilinear fractional integrals on spaces as Morrey spaces and Lipschitz spaces.
\end{abstract}

2000 Mathematics subject classification: 42B20, 42B25, 42B35.

Keywords and phrases: Morrey space, multilinear fractional integral.

\section{Introduction}

Let $\mathbb{R}^{n}$ be the $n$-dimensional Euclidean space and $\left(\mathbb{R}^{n}\right)^{m}=\mathbb{R}^{n} \times \mathbb{R}^{n} \times \cdots \times \mathbb{R}^{n}$ be the $m$-fold product space. The multilinear fractional integral is defined by

$$
I_{\alpha, m}\left(f_{1}, \ldots, f_{m}\right)(x)=\int_{\left(\mathbb{R}^{n}\right)^{m}} \frac{f_{1}\left(y_{1}\right) \cdots f_{m}\left(y_{m}\right)}{\left|\left(x-y_{1}, \ldots, x-y_{m}\right)\right|^{m n-\alpha}} d y_{1} \cdots d y_{m},
$$

where $0<\alpha<m n, m \in \mathbb{N}$.

Obviously, the multilinear fractional integral $I_{\alpha, m}$ is a natural generalization of the classical fractional integral $I_{\alpha}$. Kenig and Stein [6] as well as Grafakos and Kalton [3] considered the boundedness of a family of related fractional integrals. The main purpose of this paper is to establish some endpoint estimates for the multilinear fractional integral. Before stating our results, let us first introduce some notation.

We first recall the definition of the Morrey space [7]. For $1 \leq q \leq p<\infty$, the Morrey space $M_{q}^{p}\left(\mathbb{R}^{n}\right)$ defined as the sets of functions $f(x) \in L_{\mathrm{loc}}^{q}\left(\mathbb{R}^{n}\right)$ such that

$$
\|f\|_{M_{q}^{p}\left(\mathbb{R}^{n}\right)}:=\sup _{x_{0} \in \mathbb{R}^{n}} \sup _{r>0} \frac{1}{r^{n / q-n / p}}\left(\int_{B\left(x_{0}, r\right)}|f(y)|^{q} d y\right)^{1 / q}<\infty,
$$

where $B\left(x_{0}, r\right)$ denotes the ball in $\mathbb{R}^{n}$ with center $x_{0}$ and radius $r$.

The research was supported by the NNSF (10401002) and NNSF (10371004) of China.

(c) 2008 Australian Mathematical Society 1446-7887/08 \$A2.00+0.00 
REMARK. It is easy to see that the relation $M_{q_{1}}^{p}\left(\mathbb{R}^{n}\right) \subset M_{q_{2}}^{p}\left(\mathbb{R}^{n}\right)$ holds with $1 \leq$ $q_{2} \leq q_{1} \leq p<\infty$, and $M_{p}^{p}\left(\mathbb{R}^{n}\right)=L^{p}\left(\mathbb{R}^{n}\right)$. In addition, we know that $L^{p, \infty}\left(\mathbb{R}^{n}\right)$ is contained in $M_{q}^{p}\left(\mathbb{R}^{n}\right)$ with $1 \leq q<p<\infty$ (see [5, Lemma 1.7]). More precisely, $\|f\|_{M_{q}^{p}\left(\mathbb{R}^{n}\right)} \leq C\|f\|_{L^{p, \infty}\left(\mathbb{R}^{n}\right)}$ with $1 \leq q<p<\infty$, here and in what follows, the letter $C$ will denote a constant, not necessarily the same in different occurrences, and let $p^{\prime}$ satisfy $1 / p+1 / p^{\prime}=1$ with $p \geq 1$.

We say that $f$ belongs to weak Morrey space $W M_{q}^{p}(\Omega)$ if $1 \leq q \leq p<\infty$,

$$
\begin{aligned}
\|f\|_{W M_{q}^{p}(\Omega)} & :=\sup _{x_{0} \in \mathbb{R}^{n}} \sup _{r>0} \frac{1}{r^{n / q-n / p}} \sup _{\lambda>0}\left(\lambda^{q}\left|\left\{x \in B\left(x_{0}, r\right) \cap \Omega:|f(x)|>\lambda\right\}\right|\right)^{1 / q} \\
& <\infty .
\end{aligned}
$$

Let $0 \leq \beta<1 / n$, we say that $f \in L_{\mathrm{loc}}\left(\mathbb{R}^{n}\right)$ belongs to $\mathcal{L}(\beta)$ if there exists some constant $C_{1}$ such that for any ball $B$,

$$
\frac{1}{|B|^{1+\beta}} \int_{B}\left|f(x)-m_{B}(f)\right| d x \leq C_{1},
$$

where $m_{B}(f)=(1 /|B|) \int_{B} f(x) d x$. The smallest constant $C_{1}$ will be denoted by $\|f\|_{\mathcal{L}(\beta)} ;$ see [7].

Let us observe that for $\beta=0$, the space $\mathcal{L}(\beta)$ coincides with the version $B M O\left(\mathbb{R}^{n}\right)$ space. Moreover, $\mathcal{L}(\beta)$ coincides with $\operatorname{Lip}(\beta)$ (Lipschitz integral space) when $0<\beta$ $<1 / n$; see [7].

Let us now formulate our results as follows.

TheOREM 1.1. Let $m \in \mathbb{N},(m-1) n<\alpha<m n, 1 / p=1 / p_{1}+\cdots+1 / p_{m}$ and $1 / q=1 / q_{1}+\cdots+1 / q_{m}$ with $1 \leq q_{i}<p_{i}<\infty$ for $i=1, \ldots$, m. If $p=n / \alpha$, then

$$
\left\|I_{\alpha, m}\left(f_{1}, \ldots, f_{m}\right)\right\|_{B M O\left(\mathbb{R}^{n}\right)} \leq C \prod_{j=1}^{m}\left\|f_{j}\right\|_{M_{q_{j}}^{p_{j}}\left(\mathbb{R}^{n}\right)} .
$$

Theorem 1.2. Let $m \in \mathbb{N}, \quad 0<\alpha<m n, 1 / p=1 / p_{1}+\cdots+1 / p_{m}$ and $1 / q$ $=1 / q_{1}+\cdots+1 / q_{m}$ with $1 \leq q_{i}<p_{i}<\infty$ for $i=1, \ldots, m$. If $n / \alpha<p$ and $0<\alpha-n / p<1$, then

$$
\left\|I_{\alpha, m}\left(f_{1}, \ldots, f_{m}\right)\right\|_{L i p(\alpha-n / p)} \leq C \prod_{j=1}^{m}\left\|f_{j}\right\|_{M_{q_{j}}^{p_{j}}\left(\mathbb{R}^{n}\right)}
$$

In the endpoint case $p=n / \alpha$, Strichartz [9] proved the exponential integrability of $I_{\alpha, m}$ when $m=1$. The following result extends Strichartz's result to the case $m \geq 2$.

Theorem 1.3. Let $m \in \mathbb{N}, \quad 0<\alpha<m n, 1 / p=1 / p_{1}+\cdots+1 / p_{m}=\alpha / n$ with $1<p_{i}<\infty$ for $i=1, \ldots, m$. Let $B$ be a ball of radius $R$ in $\mathbb{R}^{n}$ and let $f_{j} \in L^{p_{j}}(B)$ 
be supported in $B$. Then there exist constants $k_{1}, k_{2}$ depending only on $n, m, \alpha, p$ and the $p_{j}$ such that

$$
\int_{B} \exp \left(k_{1}\left(\frac{\left|I_{\alpha, m}\left(f_{1}, \ldots, f_{m}\right)(x)\right|}{\prod_{j=1}^{k}\left\|f_{j}\right\|_{L^{p_{j}(B)}}}\right)^{n /(m n-\alpha)}\right) d x \leq k_{2} R^{n}
$$

THEOREM 1.4. Suppose that $m \in \mathbb{N}, 0<\alpha<m n, 1 / p=1 / p_{1}+\cdots+1 / p_{m}$ and $1 / q$

$=1 / q_{1}+\cdots+1 / q_{m}$ with $1<q_{i} \leq p_{i} \leq \infty$ for $i=1, \ldots, m$. Assume that $1 / s$ $=1 / q-\alpha / n$ and $p<n / \alpha$.

(a) If each $q_{i}>1$, then

$$
\left\|I_{\alpha, m}\left(f_{1}, \ldots, f_{m}\right)\right\|_{M_{s}^{p n / n-p \alpha}\left(\mathbb{R}^{n}\right)} \leq C \prod_{j=1}^{m}\left\|f_{j}\right\|_{M_{q_{j}}^{p_{j}}\left(\mathbb{R}^{n}\right)}
$$

(b) If $1 \leq q_{i}<p_{i}$ and $s(1 / q-1 / p)>q_{i}\left(1 / q_{i}-1 / p_{i}\right)$ for $i=1, \ldots, m$, then

$$
\left\|I_{\alpha, m}\left(f_{1}, \ldots, f_{m}\right)\right\|_{W M_{s}^{p n /(n-p \alpha)}\left(\mathbb{R}^{n}\right)} \leq C \prod_{j=1}^{m}\left\|f_{j}\right\|_{M_{q_{j}}^{p_{j}}\left(\mathbb{R}^{n}\right)}
$$

\section{The proofs of Theorems $\mathbf{1 . 1}$ and $\mathbf{1 . 2}$}

Proof of Theorem 1.1. Given $\vec{f}=\left(f_{1}, \ldots, f_{m}\right)$, for any ball $B=B\left(x_{0}, r\right)$, it suffices to prove that the following inequality

$$
\frac{1}{|B|} \int_{B}\left|I_{\alpha, m}(\vec{f})(x)-m_{B}\left(I_{\alpha, m}(\vec{f})\right)\right| d x \leq C \prod_{j=1}^{m}\left\|f_{j}\right\|_{M_{q_{j}}^{p_{j}}\left(\mathbb{R}^{n}\right)}
$$

holds.

Define $f_{j}^{0}=f_{j} \chi_{2 B}$ and $f_{j}^{\infty}=f-f_{j}^{0}$ for $j=1, \ldots, m$. Then

$$
\begin{aligned}
& \frac{1}{|B|} \int_{B}\left|I_{\alpha, m}(\vec{f})(x)-m_{B}\left(I_{\alpha, m}(\vec{f})\right)\right| d x \\
& \quad \leq \sum_{r_{1}, \ldots, r_{m} \in\{0, \infty\}} \frac{1}{|B|} \int_{B}\left|I_{\alpha, m}\left(f_{1}^{r_{1}}, \ldots, f_{m}^{r_{m}}\right)(x)-m_{B}\left(I_{\alpha, m}\left(f_{1}^{r_{1}}, \ldots, f_{m}^{r_{m}}\right)\right)\right| d x .
\end{aligned}
$$


First we estimate the term of the set corresponding to $r_{1}=\cdots=r_{m}=0$. Then

$$
\begin{aligned}
& \frac{1}{|B|} \int_{B}\left|I_{\alpha, m}\left(f_{1}^{0}, \ldots, f_{m}^{0}\right)(x)-m_{B}\left(I_{\alpha, m}\left(f_{1}^{0}, \ldots, f_{m}^{0}\right)\right)\right| d x \\
& \quad \leq \frac{C}{|B|} \int_{B}\left|I_{\alpha, m}\left(f_{1}^{0}, \ldots, f_{m}^{0}\right)(x)\right| d x \\
& \quad \leq \frac{C}{|B|} \int_{B}\left|x-y_{1}\right|^{\alpha-m n} d x \prod_{j=1}^{m} \int_{2 B}\left|f_{j}\left(y_{j}\right)\right| d y_{j} \\
& \quad \leq C \prod_{j=1}^{m}\left\|f_{j}\right\|_{M_{q_{j}}^{p_{j}}\left(\mathbb{R}^{n}\right)}
\end{aligned}
$$

since $0<m n-\alpha<n$.

Consider first the case where exactly $l$ of the $r_{j}$ are $\infty$ for some $1 \leq l<m$. We only give the arguments for one of these cases. The rest are similar and can easily be obtained from the argument below by permuting the indices. We now estimate the term

$$
\begin{aligned}
I:=\mid & I_{\alpha, m}\left(f_{1}^{\infty}, \ldots, f_{l}^{\infty}, f_{l+1}^{0}, \ldots, f_{m}^{0}\right)(x) \\
& \quad-I_{\alpha, m}\left(f_{1}^{\infty}, \ldots, f_{l}^{\infty}, f_{l+1}^{0}, \ldots, f_{m}^{0}\right)(y) \mid .
\end{aligned}
$$

In fact, let $\alpha=\sum_{i=1}^{l} \alpha_{i}$ and $0<\alpha_{i}<(m n+1) / l-n\left(1-1 / p_{i}\right)$ for $i=1, \ldots, m$. For any $x, y \in B$, then

$$
\begin{aligned}
|I| \leq & C \prod_{j=l+1}^{m} \int_{2 B}\left|f_{j}\left(y_{j}\right)\right| d y_{j} \int_{\left(\mathbb{R}^{n}\right)^{l}} \frac{|x-y|}{\left|\left(x-y_{1}, \ldots, x-y_{m}\right)\right|^{m n-\alpha+1}} \\
& \times \prod_{k=1}^{l} f_{k}^{\infty}\left(y_{k}\right) \mid d y_{1} \cdots d y_{l} \\
\leq & C r \prod_{j=l+1}^{m} r^{n\left(1-1 / p_{j}\right)}\left\|f_{j}\right\|_{M_{q_{j}}^{p_{j}}\left(\mathbb{R}^{n)}\right)} \prod_{k=1}^{l} \int_{\mathbb{R}^{n} \backslash 2 B} \frac{\left|f_{k}\left(y_{k}\right)\right|}{\left|x-y_{k}\right|^{(m n+1) / l-\alpha_{k}}} d y_{k} \\
\leq & C \prod_{j=1}^{m}\left\|f_{j}\right\|_{M_{q_{j}}^{p_{j}}\left(\mathbb{R}^{n}\right)^{n}} .
\end{aligned}
$$

It remains to estimate the last term

$$
I I:=\left|I_{\alpha, m}\left(f_{1}^{\infty}, \ldots, f_{m}^{\infty}\right)(x)-I_{\alpha, m}\left(f_{1}^{\infty}, \ldots, f_{m}^{\infty}\right)(y)\right|
$$


Let $\alpha=\sum_{i=1}^{m} \alpha_{i}$ with $\alpha_{i}=n / p_{i}$ for $i=1, \ldots, m$. For any $x, y \in B$, then

$$
\begin{aligned}
|I I| & \leq \int_{\left(\mathbb{R}^{n}\right)^{m}} \frac{|x-y|}{\left|\left(x-y_{1}, \ldots, x-y_{m}\right)\right|^{m n-\alpha+1}} \prod_{j=1}^{m} f_{j}^{\infty}\left(y_{j}\right) \mid d y_{1} \cdots d y_{l} \\
& \leq C r \prod_{j=1}^{m} \int_{\mathbb{R}^{n} \backslash 2 B} \frac{\left|f_{j}\left(y_{j}\right)\right|}{\left|x-y_{j}\right|^{(m n+1) / m-\alpha_{i}}} d y_{j} \\
& \leq C \prod_{j=1}^{m}\left\|f_{j}\right\|_{M_{q_{j}}^{p_{j}}\left(\mathbb{R}^{n}\right)}
\end{aligned}
$$

Combining (2.2), (2.3) and (2.4), then (2.1) holds. Thus, Theorem 1.1 is proved.

From Theorem 1.1 and Remark, we have the following result.

COROllary 2.1. Let $\alpha, p, p_{j}$ be as in Theorem 1.1, then

$$
\left\|I_{\alpha, m}\left(f_{1}, \ldots, f_{m}\right)\right\|_{B M O\left(\mathbb{R}^{n}\right)} \leq C \prod_{j=1}^{m}\left\|f_{j}\right\|_{L^{p_{j}, \infty}\left(\mathbb{R}^{n}\right)}
$$

Proof of TheOREM 1.2. For any $x, y \in \mathbb{R}^{n}$, we only need to prove

$$
\left|I_{\alpha, m}\left(f_{1}, \ldots, f_{m}\right)(x)-I_{\alpha, m}\left(f_{1}, \ldots, f_{m}\right)(y)\right| \leq C|x-y|^{\alpha-n / p} \prod_{j=1}^{m}\left\|f_{j}\right\|_{M_{q_{j}}^{p_{j}}\left(\mathbb{R}^{n}\right)}
$$

Let $B=B(x, r)$ with $r=|x-y|$. Define $f_{j}^{0}=f_{j} \chi_{2 B}$ and $f_{j}^{\infty}=f-f_{j}^{0}$ for $j=1, \ldots, m$. Then

$$
\begin{aligned}
& \left|I_{\alpha, m}\left(f_{1}, \ldots, f_{m}\right)(x)-I_{\alpha, m}\left(f_{1}, \ldots, f_{m}\right)(y)\right| \\
& \quad \leq \sum_{r_{1}, \ldots, r_{m} \in\{0, \infty\}}\left|I_{\alpha, m}\left(f_{1}^{r_{1}}, \ldots, f_{m}^{r_{m}}\right)(x)-I_{\alpha, m}\left(f_{1}^{r_{1}}, \ldots, f_{m}^{r_{m}}\right)(y)\right| .
\end{aligned}
$$

First we estimate the term of the set corresponding to $r_{1}=\cdots=r_{m}=0$. Then

$$
\begin{aligned}
& \left|I_{\alpha, m}\left(f_{1}^{0}, \ldots, f_{m}^{0}\right)(x)-I_{\alpha, m}\left(f_{1}^{0}, \ldots, f_{m}^{0}\right)(y)\right| \\
& \leq \int_{\left(\mathbb{R}^{n}\right)^{m}} \frac{\left|f_{1}^{0}\left(y_{1}\right) \cdots f_{m}^{0}\left(y_{m}\right)\right|}{\left|\left(x-y_{1}, \ldots, x-y_{m}\right)\right|^{m n-\alpha}} d y_{1} \cdots d y_{m} \\
& \quad+\int_{\left(\mathbb{R}^{n}\right)^{m}} \frac{\left|f_{1}^{0}\left(y_{1}\right) \cdots f_{m}^{0}\left(y_{m}\right)\right|}{\left|\left(y-y_{1}, \ldots, y-y_{m}\right)\right|^{m n-\alpha}} d y_{1} \cdots d y_{m} \\
& \quad:=E_{1}+E_{2} .
\end{aligned}
$$

Since the proof of $E_{1}$ is similar to $E_{2}$, we only give a proof of $E_{1}$. Let $\alpha=\sum_{i=1}^{m} \alpha_{i}$ with $n / p_{i}<\alpha_{i}<n$ for $i=1, \ldots, m$. Then 


$$
\begin{aligned}
E_{1} & \leq C \prod_{j=1}^{m} \int_{2 B} \frac{\left|f_{j}\left(y_{j}\right)\right|}{\left|x-y_{j}\right|^{n-\alpha_{j}}} d y_{j} \\
& \leq C \prod_{j=1}^{m} r^{\alpha_{j}-n / p_{j}}\left\|f_{j}\right\|_{M_{q_{j}}^{p_{j}}\left(\mathbb{R}^{n}\right)} \\
& \leq C r^{\alpha-n / p} \prod_{j=1}^{m}\left\|f_{j}\right\|_{M_{q_{j}}^{p_{j}}\left(\mathbb{R}^{n}\right)} .
\end{aligned}
$$

Consider first the case where exactly $l$ of the $r_{j}$ are $\infty$ for some $1 \leq l<m$. We give the arguments for one of these cases. The rest are similar. Similar to the proof of $I$ in Theorem 1.1, let $\alpha=\sum_{i=1}^{l} \alpha_{i}$ and $0<\alpha_{i}<(m n+1) / l-n\left(1-1 / p_{i}\right)$ for $i=1, \ldots, m$, then

$$
\begin{aligned}
& \left|I_{\alpha, m}\left(f_{1}^{\infty}, \ldots, f_{l}^{\infty}, f_{l+1}^{0}, \ldots, f_{m}^{0}\right)(x)-I_{\alpha, m}\left(f_{1}^{\infty}, \ldots, f_{l}^{\infty}, f_{l+1}^{0}, \ldots, f_{m}^{0}\right)(y)\right| \\
& \leq C \prod_{j=l+1}^{m} \int_{2 B}\left|f_{j}\left(y_{j}\right)\right| d y_{j} \int_{\left(\mathbb{R}^{n}\right)^{l}} \frac{|x-y|}{\left|\left(x-y_{1}, \ldots, x-y_{m}\right)\right|^{m n-\alpha+1}} \\
& \quad \times \prod_{k=1}^{l}\left|f_{k}^{\infty}\left(y_{k}\right)\right| d y_{1} \ldots d y_{l} \\
& \leq C r \prod_{j=l+1}^{m} r^{n\left(1-1 / p_{j}\right)}\left\|f_{j}\right\|_{M_{q_{j}}^{p_{j}}\left(\mathbb{R}^{n}\right)} \prod_{k=1}^{l} \int_{\mathbb{R}^{n} \backslash 2 B} \frac{\left|f_{k}\left(y_{k}\right)\right|}{\left|x-y_{k}\right|^{(m n+1) / l-\alpha_{i}}} d y_{k} \\
& \leq C r^{\alpha-n / p} \prod_{j=1}^{m}\left\|f_{j}\right\|_{M_{q_{j}}^{p_{j}}\left(\mathbb{R}^{n}\right)} .
\end{aligned}
$$

Now we estimate the last term. Let $\alpha=\sum_{i=1}^{m} \alpha_{i}$ and $0<\alpha_{i}-n / p_{i}<1 / n$ for $i=1, \ldots, m$. Then

$$
\begin{aligned}
& \left|I_{\alpha, m}\left(f_{1}^{\infty}, \ldots, f_{m}^{\infty}\right)(x)-I_{\alpha, m}\left(f_{1}^{\infty}, \ldots, f_{m}^{\infty}\right)(y)\right| \\
& \quad \leq C r \prod_{j=1}^{m} \int_{\mathbb{R}^{n} \backslash 2 B} \frac{\left|f_{j}\left(y_{j}\right)\right|}{\left|x-y_{j}\right|^{(m n+1) / m-\alpha_{i}}} d y_{j} \\
& \quad \leq C r^{\alpha-n / p} \prod_{j=1}^{m}\left\|f_{j}\right\|_{M_{q_{j}}^{p_{j}}\left(\mathbb{R}^{n}\right)} .
\end{aligned}
$$

Combining (2.6), (2.7) and (2.8), then (2.5) holds. Thus, Theorem 1.2 is proved.

We remark that when $p=\infty$ in Theorem 1.2 , the conclusion also holds. Indeed, the proof is similar to the case $p<\infty$, moreover the proof is simpler.

From Theorem 1.2 and Remark, we have the following result. 
Corollary 2.2. Let $\alpha, p, p_{j}$ be same as Theorem 1.2, then

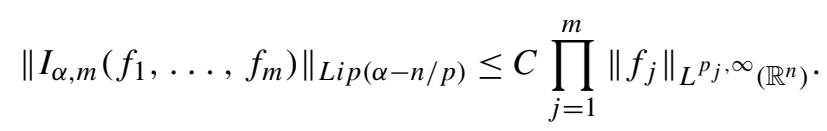

\section{The proofs of Theorems $\mathbf{1 . 3}$ and $\mathbf{1 . 4}$}

Proof of TheOREM 1.3. Let us first assume $\left\|f_{j}\right\|_{L^{p_{j}(B)}}=1, j=1, \ldots, m$, then for any $\delta>0$ and $x \in B$,

$$
\begin{aligned}
& \left|I_{\alpha, m}\left(f_{1}, \ldots, f_{m}\right)(x)\right| \\
& \leq \int_{\left|\left(x-y_{1}, \ldots, x-y_{m}\right)\right|<\delta} \frac{\left|f_{1}\left(y_{1}\right) \cdots f_{m}\left(y_{m}\right)\right|}{\left|\left(x-y_{1}, \ldots, x-y_{m}\right)\right|^{m n-\alpha}} d y_{1} \cdots d y_{m} \\
& \quad+\int_{\left|\left(x-y_{1}, \ldots, x-y_{m}\right)\right| \geq \delta} \frac{\left|f_{1}\left(y_{1}\right) \cdots f_{m}\left(y_{m}\right)\right|}{\left|\left(x-y_{1}, \ldots, x-y_{m}\right)\right|^{m n-\alpha}} d y_{1} \cdots d y_{m} \\
& \quad:=F_{1}+F_{2} .
\end{aligned}
$$

For $F_{1}$, let $\alpha=\sum_{i=1}^{m} \alpha_{i}$ with $\alpha_{i}=n / p_{i}$ for $i=1, \ldots, m$. Then

$$
\begin{aligned}
F_{1} & \leq C \prod_{j=1}^{m} \int_{\left|x-y_{j}\right|<\delta} \frac{\left|f_{j}\left(y_{j}\right)\right|}{\left|x-y_{j}\right|^{n-\alpha_{j}}} d y_{j} \\
& \leq C \prod_{j=1}^{m} \delta^{\alpha_{j}} M\left(f_{j}\right)(x) \\
& :=C_{1} \delta^{\alpha} \prod_{j=1}^{m} M\left(f_{j}\right)(x),
\end{aligned}
$$

where $M$ denotes the standard Hardy-Littlewood maximal function.

For $F_{2}$, if $\left(y_{1}, \ldots, y_{m}\right)$ satisfies $\left|x-y_{1}\right|^{2}+\cdots+\left|x-y_{m}\right|^{2} \geq \delta^{2}$, then for some $j$, say $j=m$,

$$
\left|x-y_{j}\right|=\left|x-y_{m}\right| \geq \delta / \sqrt{m} \text {. }
$$

Thus,

$$
F_{2} \leq \int_{\delta / \sqrt{m} \leq\left|x-y_{m}\right| \leq 2 R} \int_{\left(\mathbb{R}^{n}\right)^{m-1}} \frac{\left|f_{1}\left(y_{1}\right) \cdots f_{m}\left(y_{m}\right)\right|}{\left|\left(x-y_{1}, \ldots, x-y_{m}\right)\right|^{m n-\alpha}} d y_{1} \cdots d y_{m} .
$$

Define $f_{j}^{0}=f_{j} \chi_{B(x, \delta / \sqrt{m})}$ and $f_{j}^{\infty}=f-f_{j}^{0}$ for $j=1, \ldots, m$. Then 


$$
\begin{aligned}
F_{2} \leq & C \sum_{\substack{r_{1}, \ldots, r_{m} \in\{0, \infty\}\\
}} \int_{\delta / \sqrt{m} \leq\left|x-y_{m}\right| \leq 2 R} \int_{\left(\mathbb{R}^{n}\right)^{m-1}} \frac{\left|f_{1}^{r_{1}}\left(y_{1}\right) \cdots f_{m}^{r_{m}}\left(y_{m}\right)\right|}{\left|\left(x-y_{1}, \ldots, x-y_{m}\right)\right|^{m n-\alpha}} \\
& \times d y_{1} \cdots d y_{m} .
\end{aligned}
$$

We first consider the case $r_{1}=\cdots=r_{m-1}=0$. Then,

$$
\begin{aligned}
& \int_{\delta / \sqrt{m} \leq\left|x-y_{m}\right| \leq 2 R} \int_{\left(\mathbb{R}^{n}\right)^{m-1}} \frac{\left|f_{1}^{0}\left(y_{1}\right) \cdots f_{m-1}^{0}\left(y_{m-1}\right) f_{m}\left(y_{m}\right)\right|}{\left|\left(x-y_{1}, \ldots, x-y_{m}\right)\right|^{m n-\alpha}} d y_{1} \cdots d y_{m} \\
& \leq C \prod_{j=1}^{m-1} \int_{\left|x-y_{j}\right|<\delta}\left|f_{j}\left(y_{j}\right)\right| d y_{j} \int_{\delta / \sqrt{m} \leq\left|x-y_{m}\right| \leq 2 R} \frac{\left|f_{m}\left(y_{m}\right)\right|}{\left|x-y_{m}\right|^{m n-\alpha}} d y_{m} \\
& \leq C \prod_{j=1}^{m-1} \delta^{n / p_{j}^{\prime}}\left\|f_{j}\right\|_{L^{p_{j}}(B)} \int_{\delta / \sqrt{m} \leq\left|x-y_{m}\right| \leq 2 R} \frac{\left|f_{m}\left(y_{m}\right)\right|}{\left|x-y_{m}\right|^{m n-\alpha}} d y_{m} \\
& \leq C \prod_{j=1}^{m-1} \delta^{n / p_{j}^{\prime}}\left\|f_{j}\right\|_{L^{p_{j}}(B)} \delta^{\alpha-m n+n / q_{m}^{\prime}}\left\|f_{m}\right\|_{L^{p m}(B)} \\
& \leq C .
\end{aligned}
$$

Consider first the case where exactly $l$ of the $r_{j}$ are $\infty$ for some $1 \leq l<m$. We only give the argument for one of these cases. Let $\alpha=\sum_{i=1}^{m} \alpha_{i}$ with $\alpha_{i}=n / p_{i}$ for $i=1, \ldots, m$. Then

$$
\begin{aligned}
\int_{\delta / \sqrt{m} \leq\left|x-y_{m}\right| \leq 2 R} & \int_{\left(\mathbb{R}^{n}\right)^{m-1}} \frac{\left|f_{1}^{\infty}\left(y_{1}\right) \cdots f_{l}^{\infty}\left(y_{l}\right) f_{l+1}^{0}\left(y_{l+1}\right) \cdots f_{m}^{0}\left(y_{m}\right)\right|}{\left|\left(x-y_{1}, \ldots, x-y_{m}\right)\right|^{m n-\alpha}} d y_{1} \cdots d y_{m} \\
\leq & C \prod_{j=l+1}^{m-1} \delta^{n / p_{j}^{\prime}}\left\|f_{j}\right\|_{L^{p_{j}}(B)} \prod_{k=1}^{l} \int_{\delta / \sqrt{m} \leq\left|x-y_{k}\right| \leq 2 R} \frac{\left|f_{m}\left(y_{k}\right)\right|}{\left|x-y_{k}\right|^{n-\alpha_{k}}} d y_{k} \\
& \times \int_{\delta / \sqrt{m} \leq\left|x-y_{k}\right| \leq 2 R} \frac{\left|f_{m}\left(y_{m}\right)\right|}{\left|x-y_{m}\right|^{(m-l) n-\sum_{k=l+1}^{m} \alpha_{k}}} d y_{m} \\
\leq & C \prod_{j=l+1}^{m-1} \delta^{n / p_{j}^{\prime}}\left\|f_{j}\right\|_{L^{p_{j}}(B)} \prod_{k=1}^{l}\left[\log \frac{2 \sqrt{m} R}{\delta}\right]^{1 / p_{k}^{\prime}}\left\|f_{k}\right\|_{L^{p_{k}(B)}} \\
& \times \delta^{-\left[(m-l) n-\sum_{k=l+1}^{m} \alpha_{k}+n / p_{m}^{\prime}\right]}\left\|f_{m}\right\|_{L^{p_{m}(B)}} \\
\leq & C\left[\log \frac{2 \sqrt{m} R}{\delta}\right]^{\sum_{k=1}^{l} \frac{1}{p_{k}^{\prime}}} \prod_{j=1}^{m}\left\|f_{j}\right\|_{L^{p_{j}}(B)} \\
\leq & C\left[\log \frac{2 \sqrt{m} R}{\delta}\right]^{(m n-\alpha) / n}
\end{aligned}
$$


Now, we estimate the last term. Let $\alpha_{i}$ as above, then

$$
\begin{aligned}
& \int_{\delta / \sqrt{m} \leq\left|x-y_{m}\right| \leq 2 R} \int_{\left(\mathbb{R}^{n}\right)^{m-1}} \frac{\left|f_{1}^{\infty}\left(y_{1}\right) \cdots f_{m}^{\infty}\left(y_{m}\right)\right|}{\left|\left(x-y_{1}, \ldots, x-y_{m}\right)\right|^{m n-\alpha}} d y_{1} \cdots d y_{m} \\
& \leq C \prod_{j=1}^{m} \int_{\delta / \sqrt{m} \leq\left|x-y_{k}\right| \leq 2 R} \frac{\left|f_{j}\left(y_{j}\right)\right|}{\left|x-y_{j}\right|^{n-\alpha_{j}}} d y_{j} \\
& \leq C \prod_{j=1}^{m}\left[\log \frac{2 \sqrt{m} R}{\delta}\right]^{1 / q_{j}^{\prime}}\left\|f_{j}\right\|_{L^{p_{j}}(B)} \\
& \quad=C\left[\log \frac{2 \sqrt{m} R}{\delta}\right]^{(m n-\alpha) / n} .
\end{aligned}
$$

Combining (3.2), (3.3) and (3.4), we obtain

$$
F_{2} \leq C_{2}\left[\log \frac{2 \sqrt{m} R}{\delta}\right]^{(m n-\alpha) / n}
$$

Thus, by (3.1) and the above, we obtain

$$
\left|I_{\alpha, m}\left(f_{1}, \ldots, f_{m}\right)(x)\right| \leq C_{1} \delta^{\alpha} \prod_{j=1}^{m} M\left(f_{j}\right)(x)+C_{2}\left[\log \frac{2 \sqrt{m} R}{\delta}\right]^{(m n-\alpha) / n}
$$

provided $x \in B$ and $0<\delta \leq 2 \sqrt{m} R$. In particular, the choice of $\delta=2 \sqrt{m} R$ yields for all $x \in B$,

$$
\left|I_{\alpha, m}\left(f_{1}, \ldots, f_{m}\right)(x)\right| \leq C_{1} \delta^{\alpha} \prod_{j=1}^{m} M\left(f_{j}\right)(x) .
$$

Hence, the election of

$$
\delta=\delta(x)=\epsilon\left[\left|I_{\alpha, m}\left(f_{1}, \ldots, f_{m}\right)(x)\right| / C_{1} \prod_{j=1}^{m} M\left(f_{j}\right)(x)\right]^{1 / \alpha}
$$

will satisfy $\delta \leq 2 \sqrt{m} R$ for all $\epsilon \leq 1$. Now, (3.5) implies

$$
\begin{aligned}
& \left|I_{\alpha, m}\left(f_{1}, \ldots, f_{m}\right)(x)\right| \leq \epsilon^{\alpha}\left|I_{\alpha, m}\left(f_{1}, \ldots, f_{m}\right)(x)\right| \\
& \quad+C_{2}\left[\frac{1}{n} \log \left(\frac{(2 \sqrt{m} R)^{n} C_{1}^{n / \alpha}\left[\prod_{j=1}^{m} M\left(f_{j}\right)(x)\right]^{n / \alpha}}{\epsilon^{n}\left|I_{\alpha, m}\left(f_{1}, \ldots, f_{m}\right)(x)\right|^{n / \alpha}}\right)\right]^{(m n-\alpha) / n}
\end{aligned}
$$

If we use the notation $C_{3}=\left(1-\epsilon^{\alpha}\right)^{n /(m n-\alpha)}$, (3.6) is equivalent to

$$
k_{1}\left|I_{\alpha, m}\left(f_{1}, \ldots, f_{m}\right)(x)\right|^{n /(m n-\alpha)} \leq \log \left(\frac{C_{4}\left[\prod_{j=1}^{m} M\left(f_{j}\right)(x)\right]^{n / \alpha}}{\left|I_{\alpha, m}\left(f_{1}, \ldots, f_{m}\right)(x)\right|^{n / \alpha}}\right),
$$


where $k_{1}=n C_{3} / C_{1}^{n /(m n-\alpha)}$ and $C_{4}=(2 \sqrt{m} R)^{n} \epsilon^{-n} C_{1}^{n / \alpha}$. By exponentiating (3.7), we obtain

$$
\exp \left(k_{1}\left|I_{\alpha, m}\left(f_{1}, \ldots, f_{m}\right)(x)\right|^{n /(m n-\alpha)}\right) \leq \frac{C_{4}\left[\prod_{j=1}^{m} M\left(f_{j}\right)(x)\right]^{n / \alpha}}{\left|I_{\alpha, m}\left(f_{1}, \ldots, f_{m}\right)(x)\right|^{n / \alpha}}
$$

Let $B_{1}=\left\{x \in B:\left|I_{\alpha, m}\left(f_{1}, \ldots, f_{m}\right)(x)\right| \geq 1\right\}$ and $B_{2}=B \backslash B_{1}$. By (3.8),

$$
\begin{aligned}
\int_{B_{1}} \exp \left(k_{1}\left|I_{\alpha, m}\left(f_{1}, \ldots, f_{m}\right)(x)\right|^{n /(m n-\alpha)}\right) d x & \leq C_{4} \int_{B_{1}}\left(\prod_{j=1}^{m} M\left(f_{j}\right)(x)\right)^{n / \alpha} d x \\
& \leq C_{4}\left(\prod_{j=1}^{m}\left\|M\left(f_{j}\right)\right\|_{L^{p_{j}}(B)}\right)^{n / \alpha} \\
& \leq C_{5} R^{n}
\end{aligned}
$$

where $C_{5}=C(2 \sqrt{m})^{n} \epsilon^{-n} C_{1}^{n / \alpha}$. On the other hand,

$$
\int_{B} \exp \left(k_{1}\left|I_{\alpha, m}\left(f_{1}, \ldots, f_{m}\right)(x)\right|^{n /(m n-\alpha)}\right) d x \leq \exp \left(k_{1}\right)\left|B_{2}\right| \leq C_{6} R^{n}
$$

Thus, adding the integrals above over $B_{1}$ and $B_{2}$,

$$
\int_{B_{2}} \exp \left(k_{1}\left|I_{\alpha, m}\left(f_{1}, \ldots, f_{m}\right)(x)\right|^{n /(m n-\alpha)}\right) d x \leq K_{2} R^{n}
$$

Let us now turn to the general $\vec{f}=\left(f_{1}, \ldots, f_{m}\right)$. If $\left\|f_{j}\right\|_{L^{p_{j}(B)}} \neq 1$ for $j=1, \ldots, m$, then we use the notation $g_{j}=f_{j} /\left\|f_{j}\right\|_{L^{p_{j}(B)}}$ and $\vec{g}=\left(g_{1}, \ldots, g_{m}\right)$. Obviously,

$$
I_{\alpha, m}(\vec{g})=I_{\alpha, m}(\vec{f}) / \prod_{j=1}^{m}\left\|f_{j}\right\|_{L^{p_{j}(B)}}
$$

Combining (3.9) and (3.10), we obtain

$$
\int_{B} \exp \left(k_{1}\left(\frac{I_{\alpha, m}(\vec{f})(x)}{\prod_{j=1}^{k}\left\|f_{j}\right\|_{L^{p_{j}(B)}}}\right)^{n /(m n-\alpha)}\right) d x \leq k_{2} R^{n},
$$

which is our assertion. Thus, Theorem 1.3 is proved.

The proof of Theorem 1.4 is rather trivial. It is a simple consequence of Hölder's inequality on weak $L^{p}$ spaces which is a standard fact. Hence, we omit the details here. 


\section{References}

[1] F. Chiarenza and M. Frasca, 'Morrey spaces and Hardy-Littlewood maximal function', Rend. Mat. Appl. 7(3-4) (1987), 273-279.

[2] L. Grafakos, 'On multilinear fractional integrals', Studia Math. 102 (1992), 49-56.

[3] L. Grafakos and N. Kalton, 'Some remarks on multilinear maps and interpolation', Math. Ann. 319 (2001), 151-180.

[4] L. Grafakos and R. Torres, 'Multilinear Calderón-Zygmund theory', Adv. Math. 165 (2002), 124164.

[5] H. Kozono and M. Yamazaki, 'Semilinear heat equations and the Navier-Stokes equation with distributions in new function spaces as initial data', Comm. Partial Differential Equations 19 (1994), 959-1014.

[6] C. Kenig and E. Stein, 'Multilinear estimates and fractional integration', Math. Reseach. Letter 6 (1999), 1-15.

[7] J. Peetre, 'On the theory of $L^{p, \lambda}$ spaces', J. Funct. Anal. 4 (1969), 71-87.

[8] E. M. Stein, Harmonic Analysis: Real-Variable Methods, Orthogonality, and Oscillatory Integrals (Princeton University Press, Princeton, NJ, 1993).

[9] R. S. Strichartz, 'A note on Trudinger's extension of Sobolev's inequalities', Indiana Univ. Math. J. 21 (1972), 841-842.

LIN TANG, LMAM, School of Mathematics and Sciences, Peking University, Beijing, 100871, China

e-mail: tanglin@math.pku.edu.cn 